\title{
Activation of EphA4 induced by EphrinA1 exacerbates disruption of the blood-brain barrier following cerebral ischemia-reperfusion via the Rho/ROCK signaling pathway
}

\author{
FANGBIN CHEN $^{1 *}$, ZHIYANG LIU ${ }^{1 *}$, WEI PENG ${ }^{2}$, ZHIQIN GAO $^{1}$, HUI OUYANG $^{1}$, TONGJUN YAN ${ }^{1}$, \\ SONGBAI DING ${ }^{1}$, ZHANKUI CAI $^{1}$, BIN ZHAO ${ }^{1}$, LONGJIN MAO ${ }^{1}$ and ZHIYONG CAO $^{1}$ \\ ${ }^{1}$ Institute of Psychiatry, The 102nd Hospital of PLA, Changzhou, Jiangsu 213003; \\ ${ }^{2}$ Department of Psychiatry, The 92nd Hospitial of PLA, Nanping, Fujian 353000, P.R. China
}

Received December 8, 2017; Accepted June 11, 2018

DOI: $10.3892 /$ etm.2018.6460

\begin{abstract}
Vascular dementia (VD) is a syndrome characterized by progressive cognitive decline. According to previous studies, stroke is considered to be a risk factor for VD. The disruption of the blood-brain barrier (BBB) is pivotal to the pathology of stroke, as it contributes to post-stroke inflammation and edema. It has been reported that the Eph/Ephrin signaling pathway serves an important role in central nervous system injury. However, the role of EphrinA1/EphA4 signaling in BBB damage following ischemic stroke has not yet been reported. Oxygen-glucose deprivation/reperfusion was performed to detect changes in EphrinA1 and EphA4 expression in human brain microvascular endothelial cells (HBMECs). Male mice were randomly divided into four groups [Sham, ischemia-reperfusion (I/R), I/R+EphrinA1 and I/R+EphA4] to observe the role of EphrinA1 and EphA4 under $\mathrm{I} / \mathrm{R}$ conditions in vivo. The results of the present study revealed that the expression of EphrinA1 and EphA4 was significantly increased following $\mathrm{I} / \mathrm{R}$ in vitro and in vivo. The administration of soluble ligand EphrinA 1 enhanced $\mathrm{CD}^{2} 8^{+}$cell accumulation, brain edema and dysfunction of the BBB, with lower expression levels of zonula occludens-1 (ZO-1) and Claudin-5. In addition, EphrinA1-treated mice had a higher level of caspase-3 and a lower level of phosphorylated-protein kinase B. However, the effects of EphrinA1 were abolished by EphA4-Fc, an inhibitor
\end{abstract}

Correspondence to: Dr Zhiyong Cao, Institute of Psychiatry, The 102nd Hospital of PLA, 55 Heping North Road, Changzhou, Jiangsu 213003, P.R. China

E-mail: changzhoujfj102@sina.com

Abbreviations: BBB, blood-brain barrier; VD, vascular dementia; HBMEC, human brain microvascular endothelial cells; TJ, tight junctions; MCAO, middle cerebral artery occlusion

*Contributed equally

Key words: vascular dementia, stroke, ephrin, Eph Receptor, blood-brain barrier of EphA4. These results suggested that EphrinA1 exerted its effects on I/R injury via the activated EphA4 receptor. In addition, EphrinA1 decreased ZO-1 and Claudin-5 expression through the Rho/Rho associated kinase (ROCK) signaling pathway, which was attenuated by the pharmacological inhibition of Rho (C3 transferase) or ROCK (Y-27632). In conclusion, the present study provides evidence that the activation of EphA4 induced by EphrinA1 contributes to BBB damage following ischemic stroke through the Rho/ROCK signaling pathway, which highlights a potential therapeutic strategy for ischemic stroke and may help the development of preventative interventions for VD.

\section{Introduction}

Vascular dementia (VD) is one of the most prevalent forms of dementia, which progressively affects cognitive abilities due to reduced blood flow in the brain (1-4). In general, VD patients often exhibit some cognitive disorder, such as forgetfulness, depression, anxiety or disorientation (5). Unfortunately, the molecular mechanism of VD remains unidentified at present. Notably, it has been accepted that ischemic stroke is an important risk factor of VD, leading to increased mortality and morbidity (6-9). Ischemic stroke can generate blood-brain barrier (BBB) damage and brain vasogenic edema. The process includes oxidative stress, inflammation, apoptosis and excitotoxicity (10-12). Subsequent cognition impairment caused by BBB damage induced by stroke has been observed (13). Therefore, the suppression of BBB damage may help to prevent ischemic stroke and post-stroke dementia.

The BBB is the major site of blood-central nervous system exchange at the level of the brain microvessel endothelium $(14,15)$. Brain microvascular endothelial cells (BMECs), the primary structures responsible for BBB permeability, are connected by tight junctions (TJ) and control the ionic equilibrium in brain $(16,17)$. Increasing evidence has supported the idea that disorders of some $\mathrm{TJ}$-associated proteins promote the dysfunction of the BBB in many central nervous system diseases $(18,19)$. Loss of BBB integrity is one of the key events in ischemic stroke (20). Unfortunately, the exact molecular 
mechanism underlying the BBB damage associated with stroke has not been elucidated.

The Eph receptor-ligand family represents the largest family of tyrosine kinases; the interaction between Eph receptors and their ligands is pivotal to physiological functioning by regulating cell-cell contact (21). Increasing evidence has shown that the Eph-Ephrin system is involved in inflammatory processes (22). Interestingly, it has been demonstrated that EphrinA1/EphA4 signaling is pivotal to the adhesion between monocytes and endothelial cells in vitro, thus EphrinA1/EphA4 signaling may play a key role in inflammatory stimuli (23). Moreover, inflammatory processes participate in the pathology of ischemic stroke, contributing to the damage to the $\operatorname{BBB}(24,25)$. Therefore, we hypothesize that the activation of EphrinA1/EphA4 signaling promotes stroke-induced BBB damage and brain injury.

In our research, we detected the effect of EphrinA1/EphA4 signaling on BBB damage following cerebral ischemia-reperfusion (I/R) in mice. We first demonstrated that EphrinA1/EphA4 signaling could enhance ischemic brain injury via the Rho/ROCK pathway in vivo, which was associated with increased BBB permeability, as indicated by down-regulation of TJ-associated proteins and enhanced inflammatory cell infiltration.

\section{Materials and methods}

Reagents. Primary antibodies targeting EphrinA1, EphA4, Phospho-ROCK, ROCK, CD68, ZO-1 and Claudin-5 were obtained from Abcam (Cambridge, MA, USA). Recombinant Mouse EphrinA1 protein and EphA4-Fc were purchased from R\&D Systems, Inc. (Minneapolis, MN, USA). Primary antibodies targeting caspase 3 , Phospho-AKT and AKT were purchased from Beyotime Institute of Biotechnology (Shanghai, China). The primary antibody targeting $\beta$-actin was purchased from Sigma-Aldrich (Merck KGaA, Darmstadt, Germany).

Cell culture. Human brain microvascular endothelial cells (HBMECs), which retain key features of cerebral endothelial function were obtained from Cell Systems Corporation (cat. no. ACBRI376; Kirkland, WA, USA) and used for the in vitro assay. The cells were maintained in DMEM supplemented with $10 \%$ fetal bovine serum (Gibco; Thermo Fisher Scientific, Inc., Waltham, MA, USA), $100 \mathrm{IU} / \mathrm{ml}$ penicillin, $100 \mathrm{IU} / \mathrm{ml}$ streptomycin and $5 \% \mathrm{CO}_{2}$ in a humidified incubator at $37^{\circ} \mathrm{C}$.

In vivo stroke model. In this study, the mouse model of stroke was induced via the method of middle cerebral artery occlusion (MCAO), as previously described (26). All experiments were approved by the Medical Ethics Committee of the PLA 102nd Hospital for Animal Care and Use. A total of 40 male mice (20-24 g) were used to detect the effect of the EphrinA1/EphA4 signaling pathway in I/R injury. These mice were divided into four groups: Sham $(n=10)$, MCAO $(n=10)$, $\mathrm{MCAO}+$ EphrinA1 $(\mathrm{n}=10)$ and $\mathrm{MCAO}+$ EphrinA1+EphA4-Fc $(n=10)$. In the MCAO groups, temporary left MCAO was performed for $60 \mathrm{~min}$; the mice were anesthetized using 5\% chloral hydrate $(400 \mathrm{mg} / \mathrm{kg})$, and then a midline ventral neck incision was made. A 6-0 nylon monofilament (0.20-0.22 mm tip) was used to obstruct the artery lumen, followed by reperfusion for $72 \mathrm{~h}$ in the MCAO groups. In the sham group, arteries were visualized but not disturbed. The mice in the $72 \mathrm{~h} \mathrm{I} / \mathrm{R}$ groups were assigned to receive a caudal vein infusion of EphrinA1 (2 mg/kg/d) or EphA4-Fc $(1 \mathrm{mg} / \mathrm{kg} / \mathrm{d})$ at $1 \mathrm{~h}$ after reperfusion. In addition, a total of 40 male mice (20-24 g) were used to detect the effect of the Rho/Rock signaling pathway in EphrinA1/EphA4 system-mediated I/R injury; these mice were divided into five groups: Sham $(n=8)$, MCAO $(n=8)$, $\mathrm{MCAO}+$ EphrinA1 $(\mathrm{n}=8), \mathrm{MCAO}+\mathrm{Ephrin} \mathrm{A} 1+\mathrm{C} 3$ transferase $(n=8)$ and $\mathrm{MCAO}+$ EphrinA1+Y-27,632 $(\mathrm{n}=8)$. Rho inhibitor (C3 transferase) $(4 \mathrm{mg} / \mathrm{kg} / \mathrm{d})$ or ROCK inhibitor (Y-27,632) $(5 \mathrm{mg} / \mathrm{kg} / \mathrm{d})$ was infused via the caudal vein, with the first administration at 30 min prior to EphrinA1 after reperfusion. All samples in this study were collected at $72 \mathrm{~h}$ after reperfusion.

Western blotting. The harvested cortical tissues at 24, 48 and $72 \mathrm{~h}$ after MCAO were homogenized, then lysed in RIPA buffer (Beyotime Institute of Biotechnology, Shanghai, China) containing $1 \%$ protease inhibitor (Thermo Fisher Scientific, Inc.). After collecting the protein, the protein levels were quantified using a BCA protein assay kit (Thermo Fisher Scientific, Inc.). Subsequently, $50 \mu \mathrm{g}$ proteins were loaded, electrophoresed and transferred onto polyvinylidene difluoride membranes, which were blocked for $1.5 \mathrm{~h}$ using $5 \%$ milk in TBST. The membranes were incubated with primary antibodies overnight at $4^{\circ} \mathrm{C}$. After three washes, IgG-HRP secondary antibody (1:5,000 dilution) (Zhonshan Biology Company, Beijing, China) incubation was conducted for $1 \mathrm{~h}$ at $37^{\circ} \mathrm{C}$. Finally, the membranes were stained with DAB mixed liquid (Bio-Rad Laboratories, Inc., Hercules, CA, USA), and scanned using a gel imaging system.

Glucose deprivation. Oxygen-glucose deprivation/reperfusion (OGD/R) was performed to simulate ischemic conditions in vitro, as previously described (27). The HBMEC cultures were subjected to OGD/R conditions by replacing the normal medium with glucose-free Locke's solution, and were incubated under $94 \% \mathrm{~N}_{2} / 5 \% \mathrm{CO}_{2} / 1 \% \mathrm{O}_{2}$ at $37^{\circ} \mathrm{C}$ for $6 \mathrm{~h}$. The cells were then reperfused by placing them in fresh normal DMEM under normal atmospheric conditions for 12 or $24 \mathrm{~h}$.

Histological analysis. Brains were collected from all groups and fixed for histological analysis. The whole brains were extracted and treated with formalin for $24 \mathrm{~h}$. After dehydration, each brain was embedded in paraffin and cut into $8-\mu \mathrm{m}$ sections of tissue using a histotome. After rehydration, the brain tissue sections were permeabilized with $0.3 \%$ Triton-X and blocked in $5 \% \mathrm{BSA}$ for $1 \mathrm{~h}$ at $37^{\circ} \mathrm{C}$, then incubated with EphrinA1, EphA4 and CD68 antibodies overnight at $4^{\circ} \mathrm{C}$. Following primary incubation, the sections were incubated with HRP-conjugated secondary antibodies for $1 \mathrm{~h}$ at $37^{\circ} \mathrm{C}$. The chromogenic reaction was performed using DAB mixed liquid. After dehydration and treatment with Permount ${ }^{\mathrm{TM}}$ mounting medium, micrographs were captured with a light microscope.

Measurement of brain edema. The wet-dry method was used to measure the degree of brain edema following I/R injury. 
A
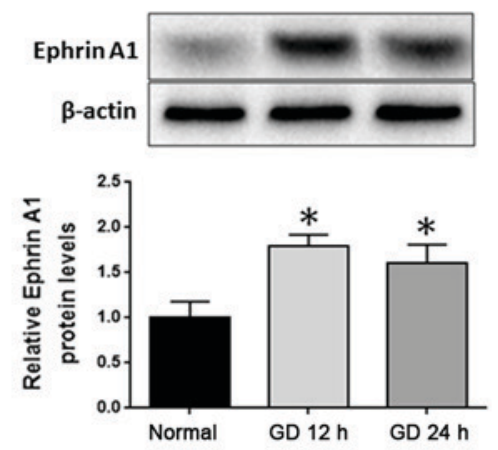

B

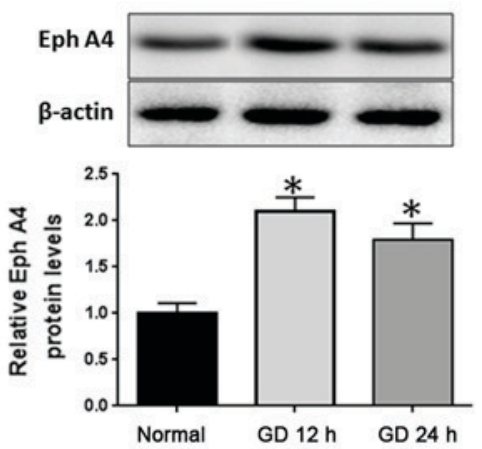

C
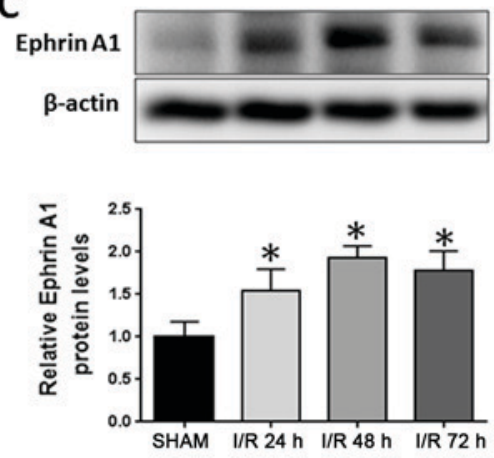

D
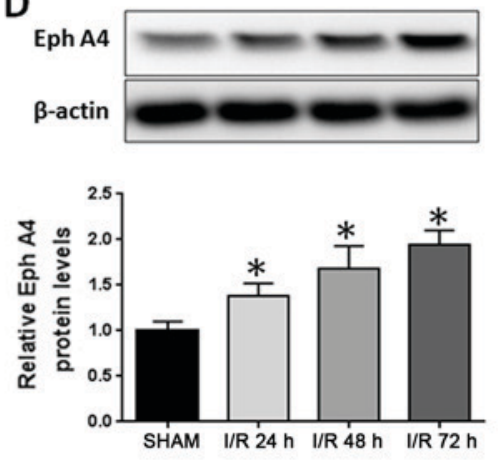

$\mathrm{E}$

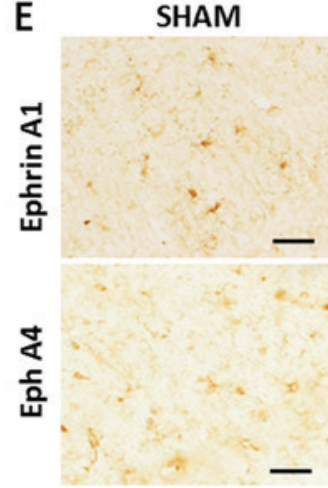

I/R $72 \mathrm{~h}$

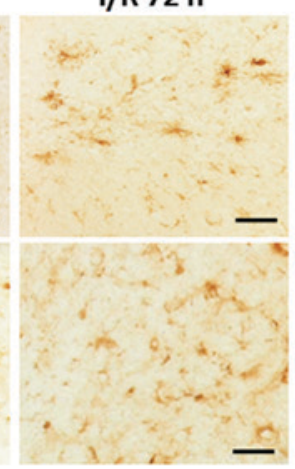

Figure 1. Expression level of EphrinA1 and EphA4 following ischemic stroke in vitro and in vivo. Expression levels of (A) EphrinA1 and (B) EphA4 in human brain microvascular endothelial cells exposed to GD conditions for 12 and 24 h, as determined via western blot analysis. Expression levels of (C) EphrinA1 and (D) EphA4 in ipsilateral cortical tissue following cerebral ischemia for 24, 48 and $72 \mathrm{~h}$, as determined via western blot analysis. (E) Representative micrographs of EphrinA1 and EphA4 in the cortex in different groups. Scale bar, $50 \mu \mathrm{m}$. Each data point represents the mean \pm standard error of the mean; $\mathrm{n}=10,{ }^{*} \mathrm{P}<0.05$ vs. the sham group. I/R, ischemia-reperfusion.

The left and right sides of the brains were divided, and the wet weights of the hemispheres were recorded immediately. Subsequently, the hemispheres were dried in the oven for 3 days at $100^{\circ} \mathrm{C}$. Brain edema was detected by calculating the water content based on the following formula: Brain water content $(\%)=(1-$ dry weight/wet weight $) \times 100 \%$.

Neurological scores. Brain function following I/R injury was examined using a neurological deficit scoring scale. The scoring system was defined as follows: $0=$ no deficit, $1=$ failure to fully extend the contralateral forelimb, $2=$ circling to the contralateral side, $3=$ leaning to the contralateral side at rest and $4=$ no spontaneous walking. Each mouse was scored three times in a blinded manner.

Rho activation assay. Based on the instructions of the Rho activation measurement kit, HBMEC lysates were incubated with rhotekin Rho-binding peptide immobilized on agarose. Activated GTP-Rho bound to rhotekin agarose was detected by anti-Rho antibody (Santa Cruz Biotechnology, Inc., Dallas, TX, USA).

Statistical analysis. All results are expressed as the mean \pm SEM. SPSS 17.0 software (SPSS, Inc., Chicago, IL, USA) was used for analysis. One-way analysis of variance (ANOVA) was used to examine the differences in the data of each group, followed by the LSD post hoc test. Neurological deficit scores were analyzed using a non-parametric Kruskal-Wallis test. The differences were considered significant at $\mathrm{P}<0.05$.

\section{Results}

Upregulation of EphrinAl and EphA4 following cerebral I/R in vivo and in vitro. To test whether EphrinA1 and EphA4 are involved in I/R injury, the expression of EphrinA1 and EphA4 was examined in stroke models in vitro and in vivo. OGD/R was used to simulate ischemia-like conditions in vitro. After subjecting the HBMECs to OGD/R for 12 or $24 \mathrm{~h}$, western blotting demonstrated that the expression of EphA4 and EphrinA1 was significantly increased (Fig. 1A and B). Next, we analyzed EphA4 and EphrinA1 expression alterations in the ipsilateral cortical tissue following cerebral ischemia for 24, 48 and $72 \mathrm{~h}$. Western blotting showed that the expression of EphA4 and EphrinA1 was significantly increased in a time-dependent manner (Fig. 1C and D), and the immunohistochemistry staining also displayed higher levels of EphA4 and Ephrin A1 expression following cerebral I/R for $72 \mathrm{~h}$ in the ipsilateral ischemic brain regions (Fig. 1E).

EphrinAl contributes to BBB damage through the EphA4 receptor. Since stroke is caused by the breakdown of $\mathrm{BBB}$ integrity, we hypothesized that the upregulation of EphrinA1 may be involved in the breakdown of BBB integrity following I/R injury in vivo. We wanted to detect whether the weak BBB stability following I/R was caused by Ephrin A1 overexpression inducing abnormal $\mathrm{TJ}$ protein regulation. We observed that the expression of ZO-1 and Claudin-5 was decreased in EphrinA1-treated I/R mice compared with the sham and I/R groups, thus indicating that the decreased BBB 
stability in I/R mice could perhaps be due to modulation of ZO-1 and Claudin-5 by EphrinA1. To determine whether the upregulation of the EphA4 receptor was responsible for the downregulation of ZO-1 induced by EphrinA1, we also used an EphA4 receptor inhibitor, EphA4-Fc, to examine the role of the EphrinA1/EphA4 interaction in I/R mice. Surprisingly, our data showed that the downregulation of ZO-1 and Claudin-5 in I/R mice treated with EphrinA1 was reversed by the administration of EphA4-Fc, which indicated that EphrinA1 contributed to the BBB damage through the EphA4 receptor (Fig. 2A and B).

Cerebral I/R injury-induced pro-apoptotic cell death signaling and brain damage were enhanced by the EphrinAl/EphA4 signaling pathway. In order to investigate the molecular mechanisms underlying the inactivation of the EphrinA1/EphA4 signaling pathway in the I/R brain, we detected apoptotic signaling using immunoblot analysis of caspase- 3 and P-AKT. We found that the caspase-3 expression level was significantly increased in I/R mice treated with EphrinA1. We also observed that I/R mice treated with EphrinA1 had significantly lower levels of P-AKT. Interestingly, pretreatment with EphA4-Fc dramatically reduced apoptotic signaling and promoted survival signaling in $\mathrm{I} / \mathrm{R}$ mice stimulated with EphrinA1, suggesting that ischemia-induced cell death is likely due to the activation of the EphA4 receptor by EphrinA1 (Fig. 3A and B). Next, we assessed inflammation levels, brain edema and neurological scores to investigate the effect of the EphrinA1/EphA4 signaling pathway in brain damage. Our data showed that EphrinA1 enhanced the infiltration of $\mathrm{CD}^{+}{ }^{+}$cells into the ipsilateral hemisphere in $72-\mathrm{h} \mathrm{I} / \mathrm{R}$ mice (Fig. 3C). In addition, in comparison with the sham and I/R groups, EphrinA1-treated I/R mice exhibited significantly higher edema in each brain hemisphere, accompanied by lower neurological scores (Fig. 3D and E). Interestingly, these effects of EphrinA1 were significantly attenuated by EphA4-Fc, suggesting that the EphrinA1/EphA4 signaling pathway contributes to brain damage in cerebral I/R injury.

EphA4 activation by EphrinAl induces BBB damage through the Rho/ROCK pathway. It has been reported that $\mathrm{Rho/ROCK}$ signaling is positively relevant to BBB damage induced by $\mathrm{I} / \mathrm{R}$. Based on this finding, we wanted to detect whether EphrinA1 modulated RhoA activity in I/R mice. We found that RhoA activity was enhanced under the conditions of $\mathrm{I} / \mathrm{R}$, and EphrinA1 promoted RhoA activity in $\mathrm{I} / \mathrm{R}$ mice (Fig. 4A). In addition, the phosphorylation of ROCK was also strengthened by EphrinA1 (Fig. 4B). Similarly, the effects of EphrinA1 were reversed by the administration of EphA4-Fc. Furthermore, we explored whether EphrinA1 regulated the expression of ZO-1 and Claudin-5 via activation of the Rho/ ROCK signaling pathway. The mice received pretreatment with C3 transferase or Y-27632 followed by the administration of EphrinA1. The expression of ZO-1 and Claudin-5 in each group was detected by western blotting. The results demonstrated that $\mathrm{C} 3$ transferase or Y-27632 inhibited the EphrinA1-induced downregulation of ZO-1 and Claudin-5 (Fig. 4C and D). Therefore, we conclude that EphA4 activation by EphrinA1 induces BBB damage through the Rho/ROCK pathway.
A

SHAM
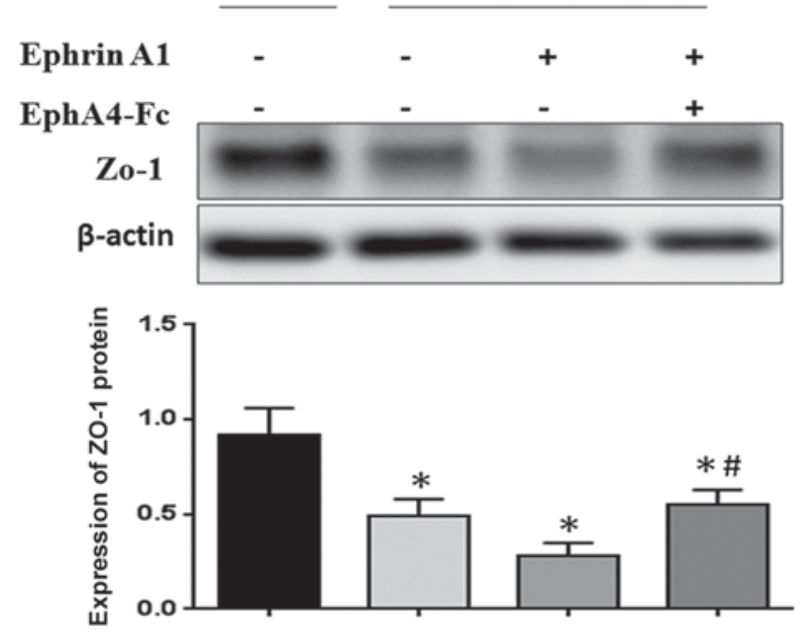

B
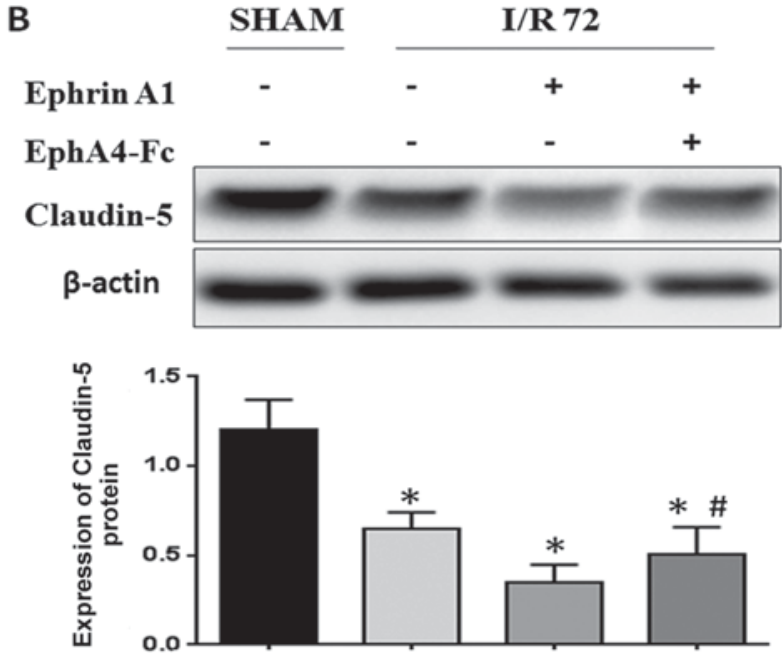

Figure 2. EphrinA1 promotes blood-brain barrier damage through the EphA4 receptor. (A) Western blot analysis revealed the expression level of (A) ZO-1 and (B) Claudin-5 in the cortex in the different groups. Bars represent the relative density against $\beta$-actin. Each data point represents the mean \pm standard error of the mean; $n=10,{ }^{*} \mathrm{P}<0.05$ vs. the sham group. ${ }^{\#} \mathrm{P}<0.05$ vs. the I/R+EphrinA1 group. ZO-1, zonula occludens-1; I/R, ischemia-reperfusion.

\section{Discussion}

Stroke is a major refractory disease with a high rate of morbidity, disability and mortality, and it significantly threatens human health and life expectancy worldwide. According to previously published statistics, stroke markedly increases the risk of dementia by 4 to 12 times (28). Additionally, post-stroke dementia increases the risk of recurrent stroke and mortality (29). At present, there is a particular need to provide an. effective therapeutic target for VD following ischemic stroke. Notably, the breakdown of the brain vascular endothelium and disorders of tight junctions induced by I/R injury lead to altered BBB permeability, which promotes brain inflammation and edema, and even cognitive impairment (30). However, the signaling mechanisms associated with I/R-induced BBB damage remain poorly understood. Previous studies have demonstrated that brain microvascular endothelial cells are an important component of the BBB; meanwhile, disorders of brain 
A
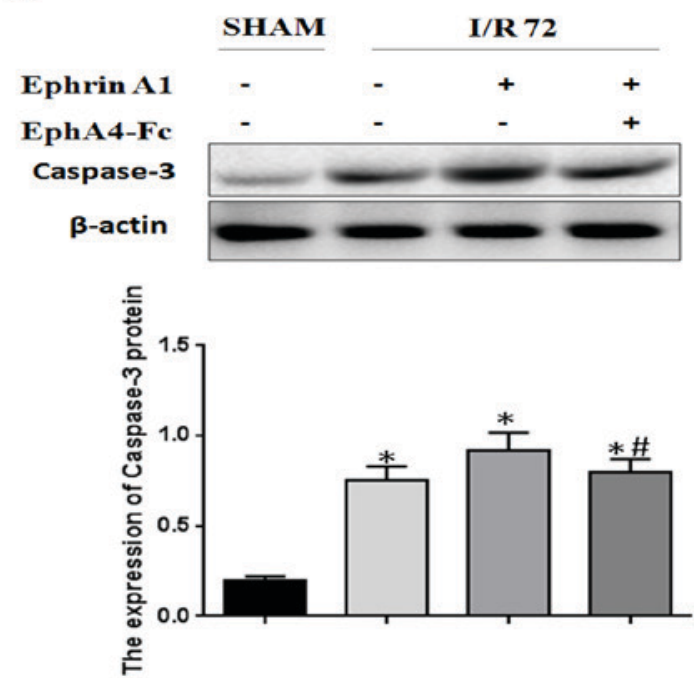

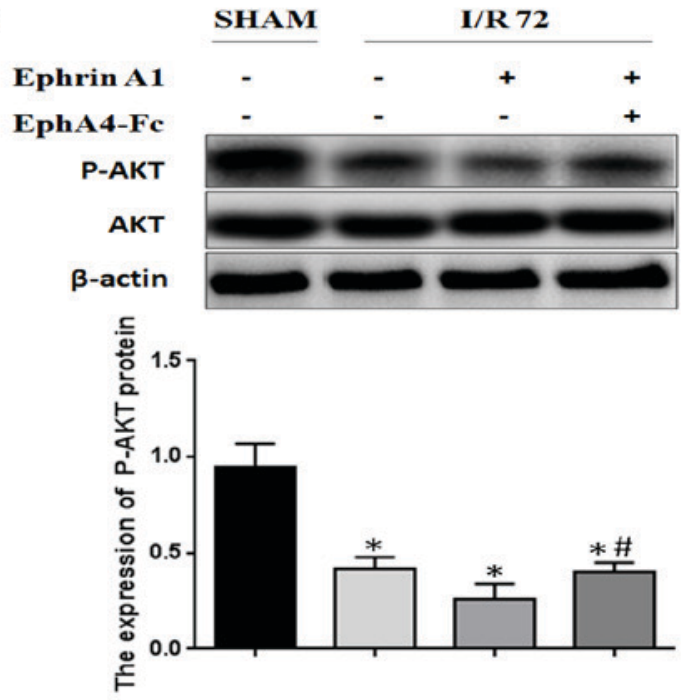

C

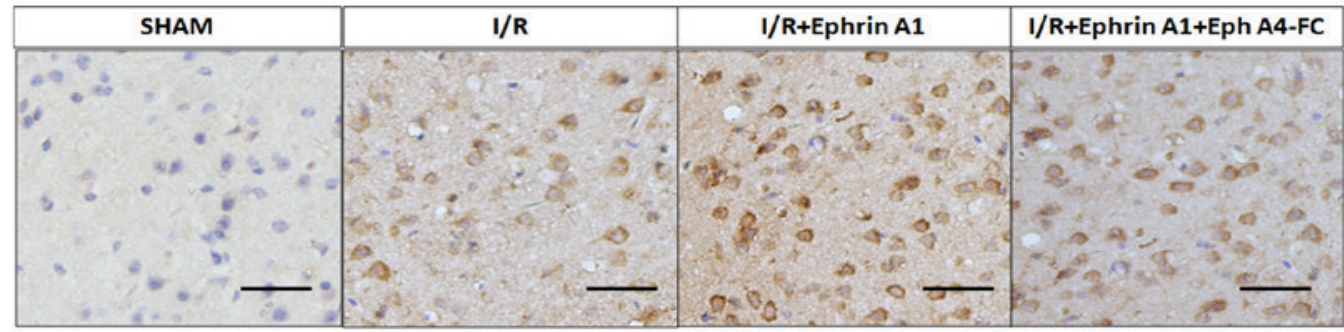

D

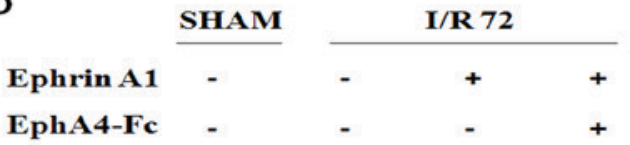

E

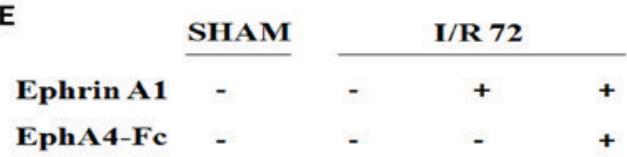

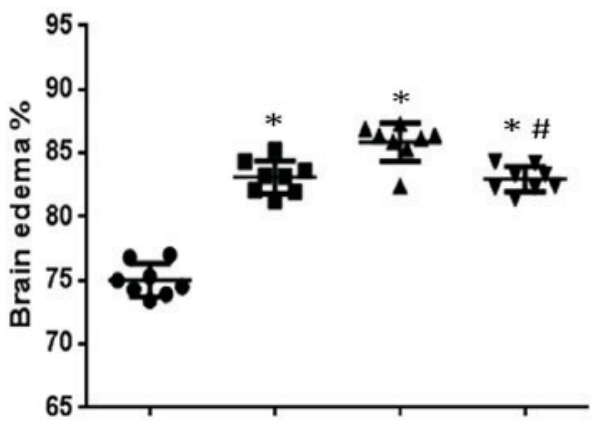

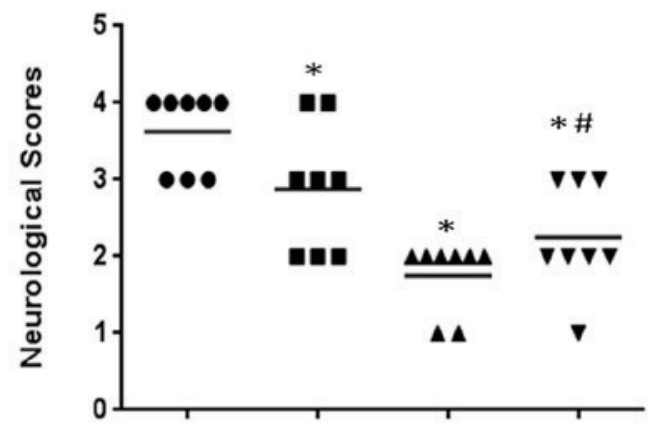

Figure 3. EphA4 activation by EphrinA1 enhances brain injury associated with increased apoptotic death, inflammatory infiltrates and brain edema, and lower functional outcomes. Western blot analysis reveals the expression of (A) caspase-3 and (B) P-AKT in the cortex. Bars represent the relative density against $\beta$-actin. (C) The expression levels of CD68 were determined by immunostaining in the cortex in the different groups. (D) Quantitative measurement of brain edema in the ipsilateral brain hemisphere using the wet-dry method. (E) Neurological scores of animals in the different groups. Scale bar, $50 \mu \mathrm{m}$. Each data point represents the mean \pm standard error of the mean; $n=10,{ }^{*} \mathrm{P}<0.05$ vs. sham group. ${ }^{*} \mathrm{P}<0.05$ vs. $\mathrm{I} / \mathrm{R}+\mathrm{Ephrin} \mathrm{A} 1$ group. $\mathrm{P}-\mathrm{AKT}$, phosphorylated-protein kinase $\mathrm{B}$; $\mathrm{CD}$, cluster of differentiation; I/R, ischemia-reperfusion.

vascular permeability following ischemic stroke contribute to secondary brain tissue damage (31). In our research, we highlighted the potential molecular mechanisms underlying BBB damage in I/R. The primary finding of this study is that the activation of the EphrinA1/EphA4 signaling pathway may enhance I/R-induced-BBB damage. Inactivation of EphrinA1/ EphA4 may be a potential treatment for ischemic stroke and post-stroke dementia.
At present, the role of the Eph/Ephrin system in the context of the pathophysiology of stroke-induced BBB breakdown and injury is unclear. It has been reported that Eph/Ephrin interactions play a key role in embryonic development (32). However, increasing studies have demonstrated that these receptors are able to promote some disease processes. Moreover, it has been proven that the activation of the EphA2 receptor by Eph ligands contributes to BBB damage and neuronal death 

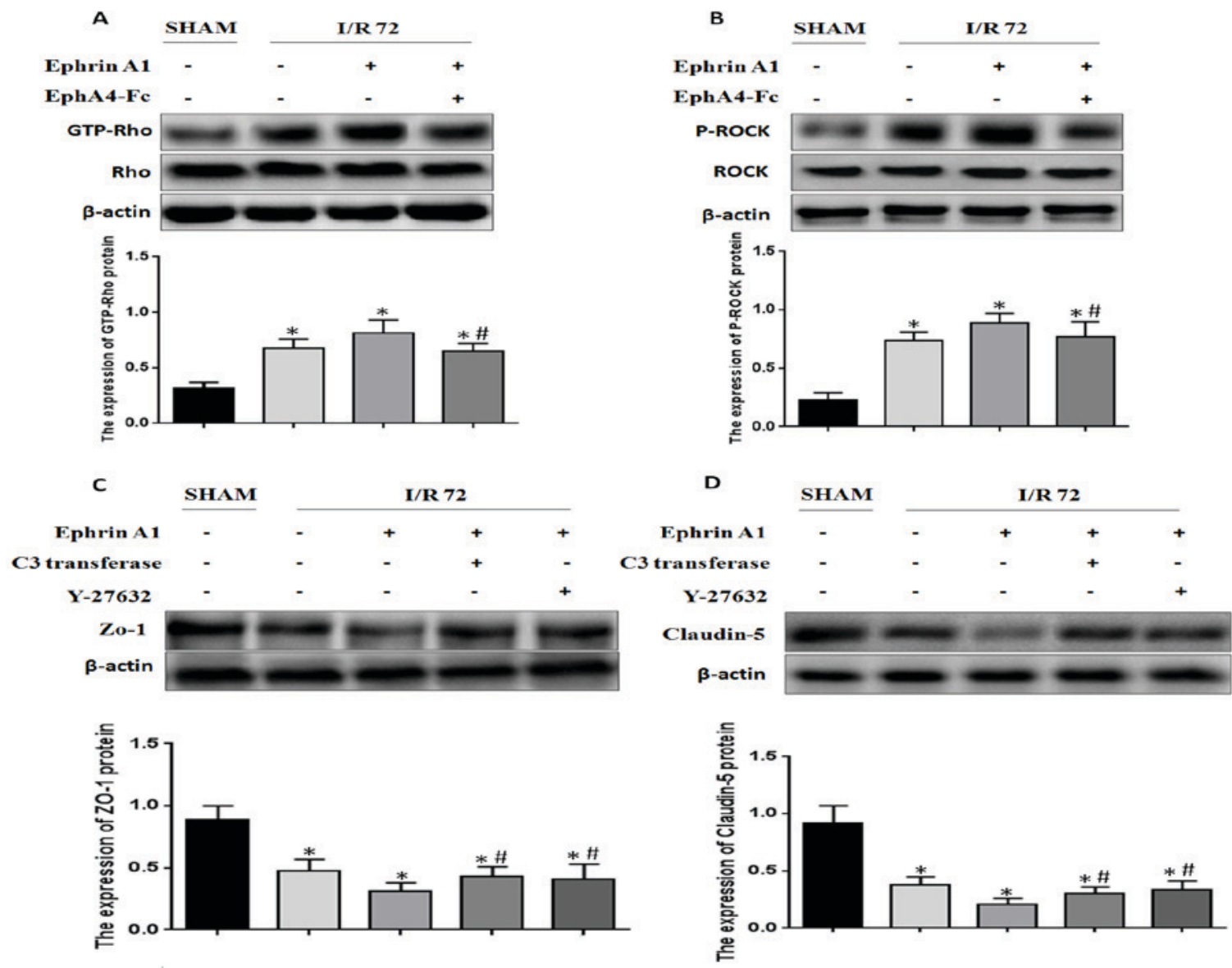

Figure 4. Activation of the EphrinA1/EphA4 signaling pathway induces blood-brain barrier damage through the Rho/ROCK signaling pathway. (A) GTP-RhoA activation was measured by a GTP-RhoA pulldown assay in the cortex. Bars represent the relative density of GTP-RhoA to total RhoA. (B) The expression of P-ROCK was determined by western blotting in the cortex. Bars represent the relative density of P-ROCK to ROCK. Western blot analysis reveals the expression of (C) ZO-1 and (D) Claudin-5 in the cortex following pretreatment with Ephrin A1, in the presence or absence of C3 transferase and Y-27632. Bars represent the relative density against $\beta$-actin. Each data point represents the mean \pm standard error of the mean; $\mathrm{n}=8$, $\mathrm{P}<0.05$ vs. the sham group. ${ }^{\sharp} \mathrm{P}<0.05$ vs. the I/R+EphrinA1 group. GTP, guanosine triphosphate; ROCK, Rho-associated kinase; P-, phosphorylated; ZO-1, zonula occludens-1; I/R, ischemia-reperfusion.

in ischemic stroke, suggesting a crucial role of Eph/Ephrin signaling in these processes (26). Furthermore, inflammatory responses participated in the complex ischemic cascade, contributing to the damage to the BBB in ischemic stroke (24). Interestingly, it has been demonstrated that EphrinA1/EphA4 signaling contributes to the adhesion of monocytes to endothelial cells in vitro, which may play a key role in inflammatory stimuli (23). Although EphrinA1 and EphA4 are known to be expressed in brain microvascular endothelial cells, little is known about whether the interaction between EphrinA1 and EphA4 plays an important role in the context of stroke-induced BBB damage and injury. Therefore, the present study aimed to evaluate the interaction between the EphA4 receptor and EphrinA1 ligand in the molecular mechanisms underlying ischemic stroke.

The data presented in this study revealed that the expression of EphrinA1 and EphA4 was upregulated, secondarily to the stimulation of GD for 12 and $24 \mathrm{~h}$. Next, these results were validated in vivo, and a similar trend in EphrinA1 and EphA4 expression was also observed after MCAO for 24, 48 and $72 \mathrm{~h}$, and the immunohistochemistry staining in the ipsilateral ischemic brain regions confirmed these results. Furthermore, we investigated the role of the interaction between EphrinA1 and EphA4 in BBB damage after stroke by evaluating the levels of the tight junction proteins ZO-1 and Claudin-5. We found that EphrinA1-treated I/R mice exhibited significantly lower levels of ZO-1 and Claudin-5 protein compared with the sham and I/R groups. However, EphA4 silencing by EphA4-Fc abolished the EphrinA1-mediated downregulation of TJ proteins. These results suggested that the upregulation of EphrinA1 contributed to BBB damage through the EphA4 receptor.

Considering that cellular apoptosis plays a key role in brain damage after ischemic stroke, we explored the effect of the EphrinA1/EphA4 signaling pathway on the levels of apoptotic proteins. Apoptosis-related proteins (Caspase-3 and $\mathrm{p}-\mathrm{Akt}$ ) were measured by western blotting to assess apoptotic signaling. Increased caspase- 3 and decreased P-AKT protein expression was observed in EprinA1-treated I/R groups compared with the sham and I/R groups, which was attenuated dramatically by pretreatment with EphA4-Fc. In addition, we hypothesized that inhibition of the EphrinA1/ EphA4 signaling pathway exhibited the potential to improve the outcome of ischemic stroke in mice. Surprisingly, we found that the EphrinA1-treated I/R mice had significantly enhanced post-stroke edema and depraved neurological outcomes when compared to sham and I/R mice, but EphA4-Fc was demonstrated to block augmented brain injury via the administration of Ephrin A1 in I/R mice. Similarly, we 
observed that activation of the EphrinA1/EphA4 signaling pathway significantly facilitated inflammatory infiltrates, which was consistent with a possible pathological mechanism in $\mathrm{I} / \mathrm{R}$ mice.

It is widely known that the Rho-kinase pathway plays important roles in many cellular functions, including contraction, motility, proliferation, and apoptosis (33). Increasing studies have reported that the excessive activity of Rho-kinase exhibits the capacity to induce oxidative stress and inflammatory response, which promotes the development of cerebrovascular diseases (34). And a previous study demonstrated that inhibition of Rho-kinase after ischemic stroke improved BBB stability (35). Next, we wanted to address whether the Ephrin A1/EphA4 interaction induced the activation of Rho and ROCK. Interestingly, we observed enhanced expression of GTP-RhoA and P-ROCK in EphrinA1-treated I/R mice, which was also attenuated by the EphA4-Fc. Finally, we analyzed whether EphrinA1/EphA4 exacerbated BBB damage via the Rho/ROCK pathway. As expected, C3-transferase and Y27632 significantly blocked the EphrinA1-mediated enhancement of ZO-1 and Claudin-5 degradation.

Our study identified the pathological effects of the EphrinA1/ EphA4 signaling pathway in ischemic stroke pathology. The overexpression of Ephrin-A1 and EphA4 contributes to the damage to the BBB and brain injury through the Rho/ROCK signaling pathway. We advocate that inhibition of the EphrinA1/ EphA4 signaling pathway in the early stages following ischemic stroke may alleviate brain damage and neuronal loss. Hopefully, the remission of ischemic stroke via the inhibition of $\mathrm{BBB}$ damage may restrain the generation and development of VD.

\section{Acknowledgements}

Not applicable.

\section{Funding}

This study was supported by a general project grant from the Nanjing Military Region (grant no. 14MS015).

\section{Availability of data and materials}

The datasets used and/or analyzed during the current study are available from the corresponding author on reasonable request.

\section{Authors' contributions}

FBC and ZYL designed the study, performed the experiments, acquired the data and wrote the manuscript. WP,ZQG, HOY and TJY helped perform the experiments in the stroke model. SBD, ZKC and BZ performed the immunohistochemistry and western blot analysis. LJM assisted with the cell culture experiments and critically reviewed the final manuscript. ZYC designed the study, wrote the manuscript and finally reviewed the manuscript.

\section{Ethics approval and consent to participate}

The present study was approved by the Medical Ethics Committee for Animal Care and Use at The 102nd Hospital of The People's Liberation Army.

\section{Consent for publication}

Not applicable.

\section{Competing interests}

The authors declare that they have no competing interests.

\section{References}

1. Plassman BL, Langa KM, Fisher GG, Heeringa SG, Weir DR, Ofstedal MB, Burke JR, Hurd MD, Potter GG, Rodgers WL, et al: Prevalence of dementia in the United States: The aging, demographics and memory study. Neuroepidemiology 29: 125-132, 2007.

2. Erkinjuntti T: Diagnosis and management of vascular cognitive impairment and dementia. J Neural Transm Suppl: 91-109, 2002.

3. Iadecola C: The pathobiology of vascular dementia. Neuron 80 : 844-866, 2013.

4. Bowler JV: Vascular cognitive impairment. Stroke 35: 386-388, 2004.

5. Nyenhuis DL and Gorelick PB: Vascular dementia: A contemporary review of epidemiology, diagnosis, prevention and treatment. J Ame Geriat Soc 46: 1437-1448, 1998.

6. Farooq MU and Gorelick PB: Vascular cognitive impairment. Curr Atheroscler Rep 15: 330, 2013

7. Jacquin A, Binquet C, Rouaud O, Graule-Petot A, Daubail B, Osseby GV, Bonithon-Kopp C, Giroud M and Béjot Y: Post-stroke cognitive impairment: High prevalence and determining factors in a cohort of mild stroke. J Alzheimers Dis 40: 1029-1038, 2014.

8. Imfeld P, Bodmer M, Schuerch M, Jick SS and Meier CR: Risk of incident stroke in patients with Alzheimer disease or vascular dementia. Neurology 81: 910-919, 2013.

9. Pendlebury ST and Rothwell PM: Prevalence, incidence and factors associated with pre-stroke and post-stroke dementia: A systematic review and meta-analysis. Lancet Neurol 8: 1006-1018, 2009.

10. Yang Y and Rosenberg GA: Blood-brain barrier breakdown in acute and chronic cerebrovascular disease. Stroke 42: 3323-3328, 2011.

11. Kamada H, Yu F, Nito C and Chan PH: Influence of hyperglycemia on oxidative stress and matrix metalloproteinase-9 activation after focal cerebral ischemia/reperfusion in rats: Relation to blood-brain barrier dysfunction. Stroke 38: 1044-1049, 2007.

12. Khatri R, McKinney AM, Swenson B and Janardhan V: Blood-brain barrier, reperfusion injury and hemorrhagic transformation in acute ischemic stroke. Neurology 79 (13 Suppl 1): S52-S57, 2012.

13. Weekman EM and Wilcock DM: Matrix metalloproteinase in blood-brain barrier breakdown in dementia. J Alzheimers Dis 49: 893-903, 2016.

14. Rochfort KD and Cummins PM: The blood-brain barrier endothelium: A target for pro-inflammatory cytokines. Biochem Soc Trans 43: 702-706, 2015.

15. Blanchette $\mathrm{M}$ and Daneman R: Formation and maintenance of the BBB. Mech Dev 138: 8-16, 2015.

16. Haseloff RF, Dithmer S, Winkler L, Wolburg H and Blasig IE: Transmembrane proteins of the tight junctions at the blood-brain barrier: Structural and functional aspects. Semin Cell Dev Biol 38: 16-25, 2015.

17. Abbott NJ, Patabendige AA, Dolman DE, Yusof SR and Begley DJ: Structure and function of the blood-brain barrier. Neurobiol Dis 37: 13-25, 2010.

18. Stamatovic SM, Keep RF and Andjelkovic AV: Brain endothelial cell-cell junctions: How to 'open' the blood brain barrier. Curr Neuropharmacol 6: 179-192, 2008.

19. Sandoval KE and Witt KA: Blood-brain barrier tight junction permeability and ischemic stroke. Neurobiol Dis 32: 200-219, 2008.

20. Lucke-Wold BP, Logsdon AF, Turner RC, Rosen CL and Huber JD: Aging, the metabolic syndrome and ischemic stroke: redefining the approach for studying the blood-brain barrier in a complex neurological disease. Adv Pharmacol 71: 411-449, 2014.

21. Lisabeth EM, Falivelli G and Pasquale EB: Eph receptor signaling and ephrins. Cold Spring Harb Perspect Biol 5: a009159, 2013.

22. Ieguchi K: Eph as a target in inflammation. Endocr Metab Immune Disord Drug Targets 15: 119-128, 2015. 
23. Jellinghaus S, Poitz DM, Ende G, Augstein A Weinert S, Stütz B, Braun-Dullaeus RC, Pasquale EB and Strasser RH: Ephrin-A1/EphA4-mediated adhesion of monocytes to endothelial cells. Biochim Biophys Acta 1833: 2201-2211, 2013

24. Gliem M, Schwaninger M and Jander S: Protective features of peripheral monocytes/macrophages in stroke. Biochim Biophys Acta 1862: 329-338, 2016

25. Moskowitz MA, Lo EH and Iadecola C: The science of stroke: Mechanisms in search of treatments. Neuron 67: 181-198, 2010.

26. Thundyil J, Manzanero S, Pavlovski D, Cully TR, Lok KZ, Widiapradja A, Chunduri P, Jo DG, Naruse C, Asano M, et al: Evidence that the EphA2 receptor exacerbates ischemic brain injury. PLoS One 8: e53528, 2013.

27. Choi JS, Kim SJ, Shin JA, Lee KE and Park EM: Effects of estrogen on temporal expressions of IL-1beta and IL-1ra in rat organotypic hippocampal slices exposed to oxygen-glucose deprivation. Neurosci Lett 438: 233-237, 2008.

28. Khedr EM, Hamed SA, El-Shereef HK, Shawky OA Mohamed KA, Awad EM, Ahmed MA, Shehata GA and Eltahtawy MA: Cognitive impairment after cerebrovascular stroke: Relationship to vascular risk factors. Neuropsychiatr Dis Treat 5: 103-116, 2009.

29. del Ser T, Barba R, Morin MM, Domingo J, Cemillan C, Pondal M and Vivancos J: Evolution of cognitive impairment after stroke and risk factors for delayed progression. Stroke 36: 2670-2675, 2005.
30. van de Haar HJ, Burgmans S, Hofman PA, Verhey FR, Jansen JF and Backes WH: Blood-brain barrier impairment in dementia: Current and future in vivo assessments. Neurosci Biobehav Rev 49: 71-81, 2015.

31. Borlongan CV, Rodrigues AA Jr and Oliveira MC: Breaking the barrier in stroke: What should we know? A mini-review. Curr Pharm Des 18: 3615-3623, 2012.

32. Hafner C, Schmitz G, Meyer S, Bataille F, Hau P, Langmann T, Dietmaier W, Landthaler M and Vogt T: Differential gene expression of Eph receptors and ephrins in benign human tissues and cancers. Clin Chem 50: 490-499, 2004.

33. Julian L and Olson MF: Rho-associated coiled-coil containing kinases (ROCK): Structure, regulation and functions. Small GTPases 5: e29846, 2014.

34. Shin HK, Salomone S and Ayata C: Targeting cerebrovascular Rho-kinase in stroke. Exp Opin Ther Targets 12: 1547-1564, 2008.

35. Gibson CL, Srivastava K, Sprigg N, Bath PM and Bayraktutan U: Inhibition of Rho-kinase protects cerebral barrier from ischaemia-evoked injury through modulations of endothelial cell oxidative stress and tight junctions. J Neurochem 129: 816-826, 2014. 